\title{
A REAL-TIME BACTERIAL BIOFILM CHARACTERIZATION PLATFORM USING A MICROFLUIDIC SYSTEM
}

\author{
S. Subramanian, Y.W. Kim, M.T. Meyer, H.O. Sintim, W.E. Bentley and R. Ghodssi * \\ University of Maryland, College Park, Maryland, USA
}

\begin{abstract}
We present a real-time multi-experiment biofilm analysis platform using a valve-actuated microfluidic system, designed to reduce growth variance of in-vitro biofilms. After 24 hours of biofilm growth in the microfluidic device the biofilms exhibited only a $9 \%$ variation in biomass, showing significantly reduced deviation in comparison to the biomass variation seen in our previous work conducted in separate microfluidic channels. A linear array charge-coupled device enabled real-time tracking of both average biomass and localized morphology by monitoring the change in optical density. We demonstrate the multi-experiment capability of the microfluidic device and the spatiotemporal monitoring capacity of the system by tracking the growth of biofilms when subjected to different treatments.
\end{abstract}

\section{INTRODUCTION}

Bacterial biofilms are the primary cause of infections in medical implants and catheters [1]. They are composed of complex communities of bacteria that are enveloped in an extracellular matrix (ECM) [1]. The presence of the ECM restricts the molecular diffusion of typical treatments like antibiotics, thereby requiring higher doses of antibiotics $(500-5000 \mathrm{x}$ in comparison to planktonic bacteria) for treatment [1,2]. The widespread use of such high doses of antibiotics for treatment is leading to the emergence of antibiotic-resistant strains. Additionally, increased ingestion of antibiotics also increases the risk of harmful side effects. Therefore, there is an urgent need for the development of new methods for the effective treatment of biofilms. However, the experimental evaluation of biofilm treatment is strongly hindered by the stochastic nature of biofilm growth [3]. Hence, it is required to develop a system that can not only facilitate multi-experiment studies for new treatment evaluation, but also enable the growth of uniform biofilms that can be used as reliable controls.

Previously, we developed microfluidic platforms that utilized simple single channel devices integrated with optical measurement methods for biofilm characterization [3, 4]. However, nonuniform biofilm growth was a key challenge in these microfluidic systems. The biomass variation observed between the single channel devices was about $25 \%$. In this work, we have developed a microfluidic platform that reduces biofilm growth variation to less than $10 \%$. This was achieved by integrating hydraulic valve actuators that were used for sectioning of biofilms grown in a horizontal channel by maintaining a single source of bacterial suspension. The detection of biofilm growth was achieved using a linear array charge-coupled device (CCD) that was used to measure the change in optical density (OD) of the biofilms. This was previously demonstrated as a reliable method for measuring the biomass of bacterial biofilms [3, 4]. The spatial resolution of the CCDs enabled real-time monitoring of both the average biomass variation and the localized biofilm morphology.

We evaluate the performance of the platform by monitoring the growth of Escherichia coli (E. coli) W3110 biofilms. The biofilm is grown in the center horizontal channel of the microfluidic reactor for 24 hours, following which the center horizontal channel is sectioned into four and each section is then subjected to different treatments for an additional 24 hours. By utilizing one of the biofilm sections as a reference, we ensure that the results of the various experiments performed on the same device are compared to a common control. The three treatments used include a traditional antibiotic (gentamicin), a small molecule inhibitor (autoinducer-2 (AI-2) analog) and a detergent (sodium dodecyl sulfate (SDS)). While antibiotics and detergents are established treatments for bacterial infections, AI-2 analogs have recently been shown to inhibit biofilms by preventing bacterial communication or quorum sensing (QS) that is critical to biofilm formation [5].

Thus, the multi-experiment capability of the microfluidic device enabled the evaluation of multiple treatments on the same biofilm while providing an integrated control. Additionally, the integration of the valved microfluidic reactor with a real-time OD monitoring system enabled high-throughput analyses on uniform biofilms and demonstrated significant reduction of biofilm growth in an analog environment. Furthermore, the unique capabilities of this microsystem can be useful in various biological studies, including new drug discovery.

\section{METHODS}

\section{Microfluidic Biofilm-Sectioning Device}

The microfluidic biofilm-sectioning device consists of a single horizontal channel that can be sectioned into multiple parts using hydraulically actuated push-down valves. The push-down valves can be operated in open or close mode through the application of pressure through the valve actuation channel. In the push-down valve configuration, a membrane between the channels is pushed down using pneumatic or hydraulic pressure, thus sealing
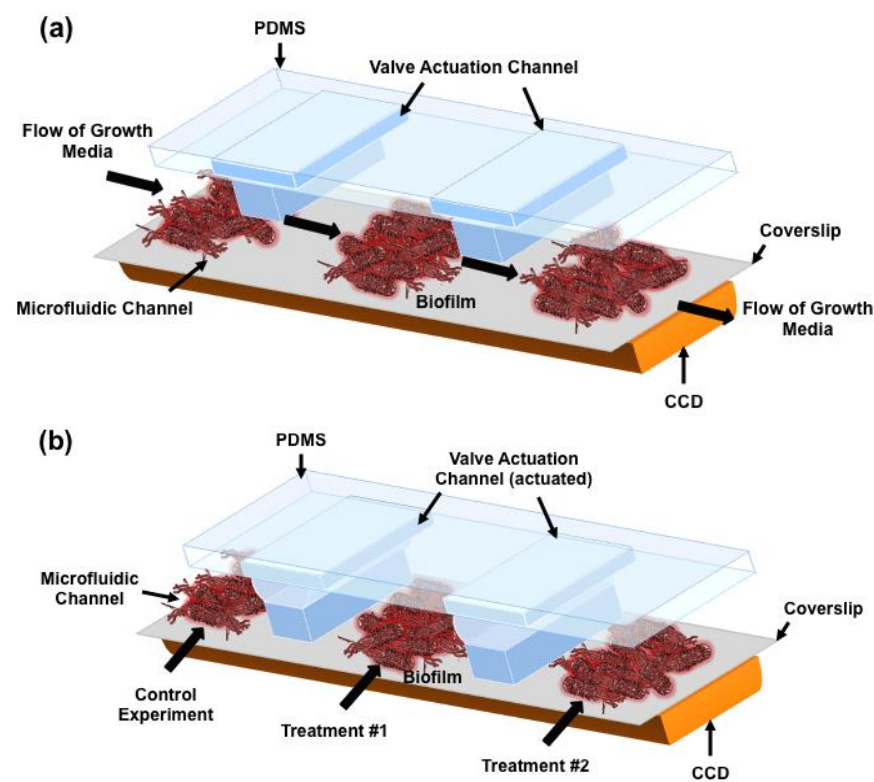

Figure 1: Schematic of platform operation. (a) Biofilm growth in the open channel, (b) Sectioning of the grown biofilms (actuated valves). The CCD monitors the biofilm growth across the length of the microfluidic channel in real-time. 
the lower microfluidic channel that contains the fluid of interest [6].

The valved microfluidic biofilm reactor is a Polydimethylsiloxane (PDMS) based two-level device. On the top is the valve actuation channel layer and on the bottom is the microfluidic channel layer. A schematic of the valve-actuated microfluidic system and the continuous OD monitoring system is shown in Figure 1. A single uniform biofilm is grown in the center horizontal channel by actuating only one set of valves known as the side valves. The biofilm is then divided into multiple sections by hydraulically actuating a second set of valves called the sectioning valves, thereby enabling multi-experiment studies on the same biofilm.

\section{Two-Depth Microfluidic Mold}

(a) SU-8 patterning on Si wafer

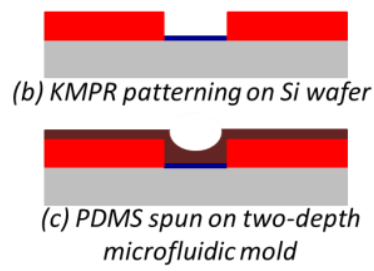

Valve Actuation Channel Mold
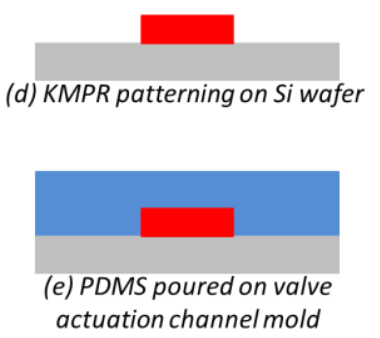

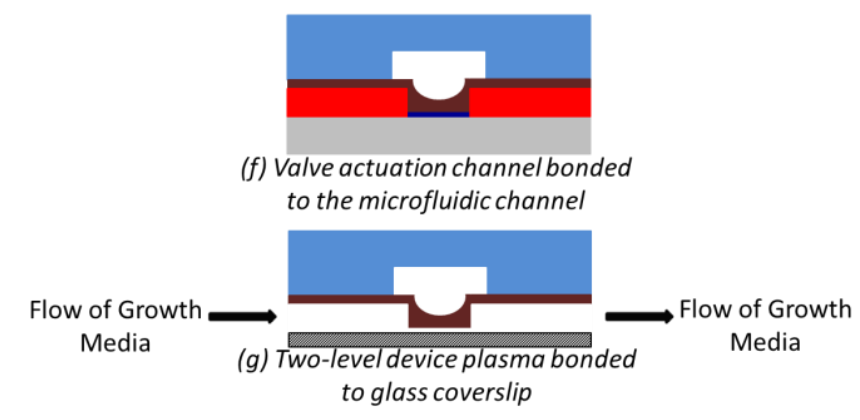

\SU8-2015 $\square$ Si $\square$ KMPR $\square$ 20:1 PDMS

5:1 PDMS $\square$ Glass coverslip

Figure 2: Fabrication process flow for the two-level valved microfluidic device. The direction of the fluid flow is along the page, from the left to the right of the microfluidic channel just above the coverslip, as indicated by the arrows. The valve actuation channel is perpendicular to the fluidic channel.

We fabricate the two-level microfluidic device using traditional multilayer soft lithography techniques. The process flow for the fabrication of the device molds and the microfluidic device are shown in Figure 2. The two-step photolithography using negative photoresists, a $20 \mu \mathrm{m}$ thick valve region using SU82015 allows for easy sealing of the valves and a $100 \mu \mathrm{m}$ tall microfluidic channel region using KMPR-1050, provides the necessary growth area for biofilms. This process allows for the patterning of a multi-depth microfluidic mold without the need for additional passivation of the first resist layer, thereby demonstrating a simplified and reliable fabrication process flow (Figure 2 (a)-(b)). The valve actuation channel mold is fabricated by patterning a single $100 \mu \mathrm{m}$ tall KMPR-1050 layer (Figure 2 (d)). Both molds can be reused to produce multiple devices. PDMS (Sylgard 184, Dow Corning, USA) in the ratio of 20:1

silicone elastomer to curing agent is spun over the two-depth microfluidic mold (Figure 2 (c)), and a ratio of 5:1 silicone elastomer to curing agent is poured over the valve actuation channel (Figure 2 (e)) and both molds are cured at $60{ }^{\circ} \mathrm{C}$ for 15 minutes. The valve actuation channel layer is peeled off the mold and the inlet ports are punched using a $2 \mathrm{~mm}$ dermatological punch. The two PDMS layers are then aligned and baked at $80^{\circ} \mathrm{C}$ for 3 hours to promote bonding between them. The final two-level device is peeled off the microfluidic channel mold and irreversibly plasma bonded to a glass coverslip. Ports for interfacing the inlets and outlets of the microfluidic channel are finally punched using the $2 \mathrm{~mm}$ dermatological hole punch. Tygon tubing is connected to the inlets and outlets of the device using a tubing coupler, and the other end of the tubing is connected to a syringe pump (KDS230, KD Scientific). The valve actuation channel is primed with water mixed with standard food coloring at a rate of $20 \mu \mathrm{l} / \mathrm{hr}$, to enable hydraulic actuation.

\section{Optical Density Monitoring Platform}

The optical density monitoring of biofilms has been previously demonstrated using the CCD devices [4]. In this work, the CCDs (TSL1402R, Texas Advanced Optoelectronic Solutions) feature $258 \times 1$ linear pixel arrays with individual photodiodes measuring $120 \mu \mathrm{m}(\mathrm{H})$ by $70 \mu \mathrm{m}(\mathrm{W})$ spaced $55 \mu \mathrm{m}$ apart, thereby spanning an overall array length of $1.6 \mathrm{~cm}$. An external diffusive edge-lit LED light panel (Luminous Film USA) was used to illuminate the platform uniformly. The wavelength of the light source was tuned to be at $630 \mathrm{~nm}$ in order to match the sensitivity of the CCDs. The PDMS microchannels that were bonded to a transparent coverslip substrate allow for light transmission to the CCD photopixels. The CCD devices were actuated by an external power supply and function generators (Agilent E3631A) and were used to control the data sampling and provide power to the electronics. The signal readout from the device was achieved using a data acquisition card (NI USB-6221, National Instruments) that was programed to record data using a Labview program.

\section{Experimental Procedure}

The CCD platform was used to continuously monitor the growth of $E$. coli $\mathrm{W} 3110$ biofilms from time $\mathrm{t}=0 \mathrm{hrs}$ to $\mathrm{t}=48 \mathrm{hrs}$, in all the sections of the microfluidic device. An overnight culture of E. coli W3110 suspension was diluted in Luria Broth (LB) media, to a final $\mathrm{OD}_{600}$ of $0.3 \mathrm{AU}$. Biofilm growth is achieved by initially seeding the center horizontal microfluidic channel (side valves actuated) with the diluted bacterial suspension for 2 hours at $37^{\circ} \mathrm{C}$ with no flow to allow for microbial attachment to the substrate. Fresh LB media is then flowed through the center horizontal channel at a rate of $10 \mu \mathrm{l} / \mathrm{hr}$ using a syringe pump for 24 hours (Figure 1a and 3a). This provides the bacteria with the necessary nutrients and environment that promotes biofilm growth. We note that the increased shear in the valve region prevents biofilm growth locally. Following the growth, the center channel was sectioned into four by actuating the sectioning valves (Figure $1 \mathrm{~b}$ and $3 \mathrm{~b}$ ). Different treatment: control (LB media, section 1), antibiotic (gentamicin, $10 \mu \mathrm{g} / \mathrm{mL}$, section 2), a small molecule biofilm growth inhibitor, (autoinducer-2 analog (AI-2 analog), $100 \mu \mathrm{M}$, section 3), and a detergent (sodium dodecyl sulfate (SDS), $0.2 \%$, section 4), were introduced into the four sections of the device for an additional 24 hours at the rate of $10 \mu \mathrm{l} / \mathrm{hr}$. The control biofilm section was provided with LB media at the same flow rate as the other sections in order to provide a reference, thereby ensuring that the results of the treatments are compared to an integrated control.

OD measurements were recorded every 8 minutes, across the length of the microfluidic channel, for each section of the device. 
These measurements enabled the monitoring of the spatio-temporal development of the bacterial biofilms in each section of the microfluidic device, both during the biofilm growth and treatment.

\section{RESULTS AND DISCUSSION}

\section{Microfluidic Valve and Device Operation}

The valves of the microfluidic device belong to one of the following two sets: 1) the side valves, and 2) the sectioning valves. Either set of valves can be actuated by application of 10 psi of pressurized nitrogen. By actuating the side valves of the device as shown in Figure 3 (a), the fluid can be directed from the inlet to the outlet of the center horizontal channel of the device, whereas actuating the sectioning valves as shown in Figure 3 (b) results in the center horizontal channel being sectioned into four sections of equal length. Thus, both sets of valves were successfully demonstrated. Therefore, using this platform, a uniform biofilm grown in the horizontal channel of the device (Figure 3a), is sectioned into four using the hydraulically actuated valves following which each section is individually treated using different methods (Figure $3 b$ ).

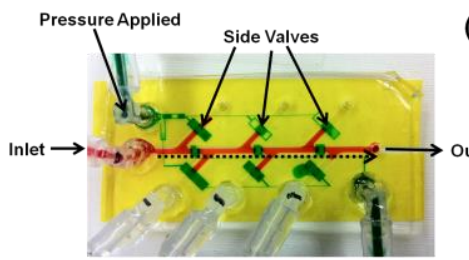

(a)

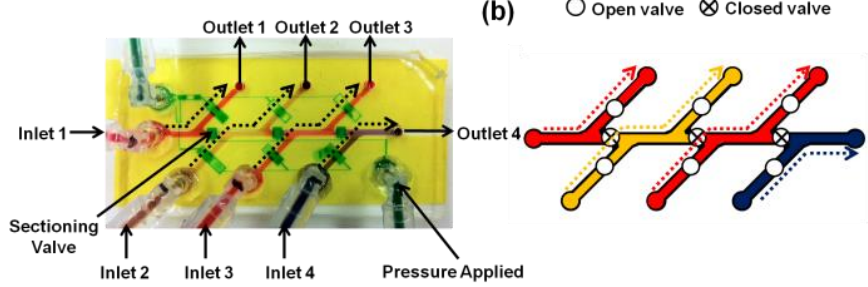

Figure 3: Device in different modes of operation. (a) Side valves actuated to allow for biofilm growth in the center channel, $(b)$ Sectioning valves actuated to allow introduction of four different treatments in four sections of the device. The four sections are shown in different colors (from left to right): section 1 (red), section 2 (yellow), section 3 (red), and section 4 (blue). The dotted arrows show the direction of fluid flow.

\section{Optical Density Monitoring}

It has been previously demonstrated that the total biomass of the biofilm can be indirectly measured by monitoring the change in OD [3, 4]. Figure 4 shows the measured average change in OD during biofilm growth in each of the four sections of the device with respect to the baseline OD measured at time $t=0$ hrs. The error bars represent the spatial variation of the biofilm across the length of the microfluidic section. After 24 hours of growth, the change in OD in each section is observed to converge to a single point. Statistical correlation was demonstrated between the OD measurements obtained for the four sections at the end of 24 hours (ANOVA, P > 0.05), thereby validating the growth of a uniform biofilm across the center channel. After 24 hours of growth, the variation in biomass between the four sections was calculated to be only $9 \%$, as compared to the $20-25 \%$ biomass variation seen in our previous work conducted in separate microfluidic channels [3]. Although a sharp increase in OD, corresponding to an increase in biomass is observed around $\mathrm{t}=18 \mathrm{hrs}$ during the biofilm growth, the
OD then rapidly decreases and converges to a single point. We hypothesize that this reduction in biomass and therefore the convergence of the OD of the four sections of the device to a single point at $\mathrm{t}=24 \mathrm{hrs}$ is possibly a result of a self-leveling effect due to the increased shear experienced by the thick biofilms in the constricted microfluidic channels.

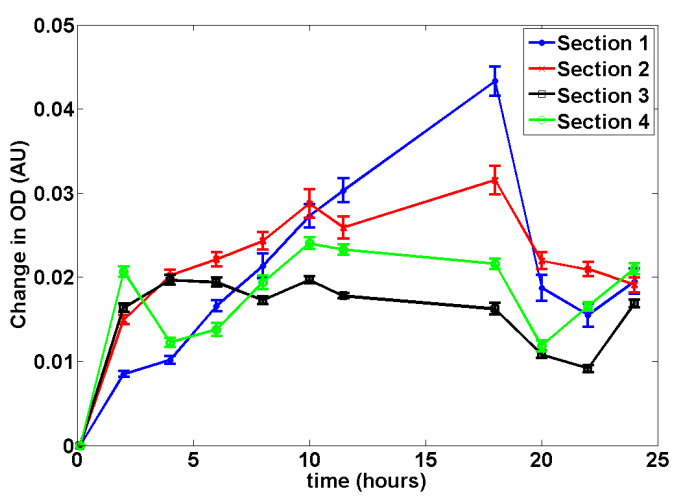

Figure 4: Measured average change in $O D$ across the length of each section $(n=162)$ at representative time points during biofilm growth. The error bars represent the spatial variance of the biofilm in each section of the channel. The variation in OD across the four sections was observed to reduce to $9 \%(P>0.05)$ after 24 hours of growth. It is hypothesized the reduction in biomass variation between sections is possibly a result of a self-leveling effect due to increased shear in constricted channels.

Figure 5 plots the change in OD during the 24-hour biofilm treatment. The three treatments, the antibiotic (gentamicin, 10 $\mu \mathrm{g} / \mathrm{mL}$ ), the small molecule biofilm growth inhibitor, (AI-2 analog, $100 \mu \mathrm{M}$ ), and the detergent (sodium dodecyl sulfate (SDS), $0.2 \%$ ) applied to sections 2,3 , and 4 respectively, showed a reduction in biofilm growth compared to the control section (pure LB media, section 1). As seen from Figure 5, the section 3 treated with the AI-2 analog treatment resulted in the slowest growth. This correlates with previously obtained results which demonstrate the efficacy of analogs to interrupt the bacterial communication in

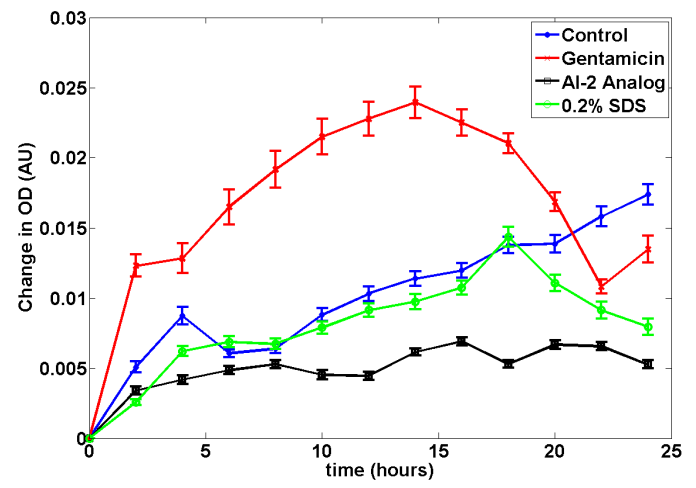

Figure 5: Measured average change in $O D$ across the length of each biofilm section $(n=162)$ at representative time points, during biofilm treatment in the four sections. The section treated with AI2 analog shows the least increase in biomass. The error bars represent the spatial variation of the biofilm in each section of the microfluidic channel. 
biofilms, also known as quorum sensing (QS) that is required for biofilm growth, thereby reducing increase of total biomass [5, 7].

The percentage relative change in average biomass was also calculated for each section of the device after the 24-hour treatment. It is calculated as the ratio of the increase in biomass after treatment to the total biomass formed at the end of the initial 24-hour biofilm growth. The equation used to calculate the percentage relative change in average biomass and the calculated values for each section are plotted in Figure 6a. As expected, the control shows the most increase in biomass, with a $166 \%$ relative increase in average biomass. Treatment with gentamicin resulted in a small decrease in biomass in comparison to the control and showed a $114 \%$ increase in average biomass. The AI- 2 analog and the detergent (SDS) resulted in the similar reduction in biomass growth with relative change in average biomass of $68 \%$ and $66 \%$

(a)

Relative Change in

Average Biomass (\%)

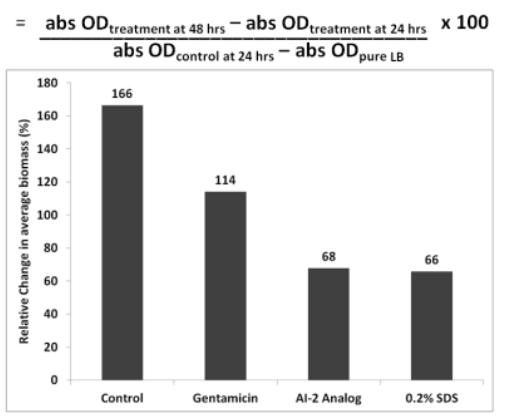

(b)

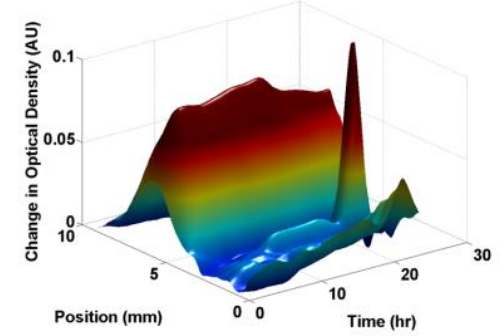

(c)

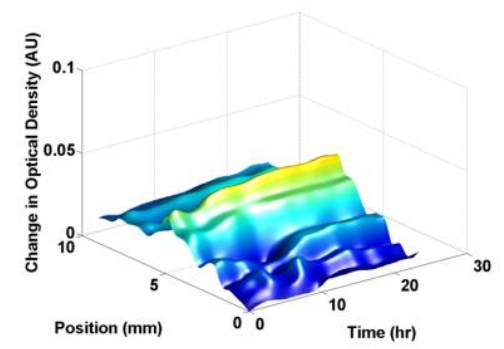

Figure 6: (a) Relative percentage change in average biomass calculated using the OD measured after the biofilm treatment in each of the four sections of the microfluidic optical platform. $3 D$ spatiotemporal plot of biofilm during treatment in $(b)$ section 1 (control) showing a significant change in $O D(\sim 0.1 \mathrm{AU})$ indicative of a large increase in biomass, and (c) section 3 (AI-2 analog treatment) showing a smaller change in $O D(\sim 0.03 \mathrm{AU})$ indicative of a less significant increase in biomass.

respectively. Specifically, the analog reduced the average biomass $88 \%$ more than the antibiotic. Additionally, Figures 6 (b) and 6 (c) demonstrates the capability of this OD based real-time monitoring system to track the localized change in biofilm morphology thereby alleviating the need for additional imaging equipment. As shown in the plots, the control (Figure 6 (b)) shows a significant change in $\mathrm{OD}(\sim 0.1 \mathrm{AU})$ indicative of a large increase in biomass, whereas the section treated with AI-2 analog (Figure 6 (c)) shows a much smaller change in OD $(\sim 0.03 \mathrm{AU})$. These spatiotemporal plots provide a 3-dimensional representation of the reduction in biofilm growth due to analog treatment, as compared to nontreated biofilms.

\section{CONCLUSIONS}

The growth of uniform biofilms using valve-actuated microfluidics for multi-experiment analyses is demonstrated in this work. The biomass variance of the biofilms grown in the microfluidic reactor was significantly lower than the biomass variation of biofilms grown in separate microfluidic channels. Using this platform, three different biofilm treatments were evaluated and compared to the integrated control. Treatment of the biofilm with AI-2 analog resulted in the slowest growth and reduced the average biomass $88 \%$ more than the antibiotic. Thus, the unique capability of this valved microfluidic biofilm reactor to section uniform biofilms combined with the real-time OD monitoring platform can facilitate high-throughput biofilm studies, including new drug discovery.

\section{ACKNOWLEDGEMENT}

The authors would like to acknowledge the Robert W. Deutsch Foundation for financial support. The authors would also like to thank the Maryland Nanocenter and its Fablab for facility support. Travel support has been generously provided by the Transducer Research Foundation.

\section{REFERENCES}

[1] J.W. Costerton, P.S. Stewart, and E.P. Greenberg, "Bacterial Biofilms: A Common Cause of Persistent Infections," Science, 21, 284 (1999).

[2] H. Anwar, M.K. Dasgupta, and J.W. Costerton, "Testing the susceptibility of bacteria in biofilms to antibacterial agents", Antimicrobial Agents and Chemotherapy, 34, 2043 (1990).

[3] M.T. Meyer, V. Roy, W.E. Bentley, and R. Ghodssi, "Development and validation of a microfluidic reactor for biofilm monitoring via optical methods," Journal of Micromechanics and. Microengineering, 21, 054023 (2011).

[4] Y.W. Kim, M.P. Mosteller, M.T. Meyer, H. Ben-Yoav, W.E. Bentley, and R. Ghodssi, "Microfluidic biofilm observation, analysis and treatment (Micro-BOAT) platform", Technical Digest of the 2012 Solid-State Sensor and Actuator Workshop, Hilton Head Isl., SC, 6/3-6/7, Transducer Research Foundation, Cleveland (2012), pp. 233-236.

[5] V. Roy, M.T. Meyer, A.I. Smith, S. Gamby, H.O. Sintim, R. Ghodssi, and W.E. Bentley, "AI-2 analogs and antibiotics: a synergistic approach to reduce bacterial biofilms", Applied Microbiology and Biotechnology, 97, 2627 (2013).

[6] V. Studer, G. Hang, A. Pandolfi, M. Ortiz, W.F. Anderson, and S.R. Quake, "Scaling properties of a low-actuation pressure microfluidic valve", Journal of Applied Physics, 95, 393 (2004).

[7] V. Roy, J.A.I. Smith, J. Wang, J.E. Stewart, W.E. Bentley, and H.O. Sintim, "Synthetic analogs tailor native AI-2 signaling across bacterial species", Journal of American Chemical Society, 132, 11141 (2010).

\section{CONTACT}

*R. Ghodssi, tel: +1-301-405-8158; ghodssi@umd.edu 